\title{
Scope of Nanotechnology in Drug Delivery
}

Kashif Maroof ${ }^{1}$, Farya Zafar ${ }^{1}$, Huma Ali', Safila Naveed ${ }^{2 *}$ and Sidra Tanwir ${ }^{1}$

${ }^{1}$ Ziauddin University Karachi, Pakistan

2Jinnah University for Women Karachi, Pakistan

\begin{abstract}
Today more than $70 \%$ of newly discovered drugs (independent from the therapeutic class) showed poor water solubility which may results a serious challenge to the development of new compounds. There are several drug formulation strategies are available to further improve the solubility and / or dissolution rate i.e. solid dispersion technique, self-emulsifying systems or complex formation. These strategies have been successfully used in the past but today nanotechnology has produced a massive impact to meet the needs. Various factors particularly economic, social, public health and environmental benefits have together made nanotechnology based drug delivery systems a very popular one. In this article we have discussed the scope of nanotechnology in the designing of new drug delivery systems.
\end{abstract}

Keywords: Nanotechnology; Self-emulsifying systems; Bioavailability

\section{Introduction}

Nanotechnology is characterized as the science, engineering and technology carried out at the nano scale [1]. It is a revolutionary technique which utilizes devices and molecules in the range of 1-100 nanometers. The use of particles in very diminutive range helps to facilitate the features and overall quality of the system. Particles exposed to nanotechnology exhibits superior characteristics including resistance to settling, higher saturation solubility state and protection from decomposition, enhanced drug release, increased adhesion to biological membranes, improved strength and reduced weight, enhanced absorption and improved chemical reactivity as compared to their larger scale corresponding item [2]. Nanotechnology tailors drugs at a molecular level which results in reducing side effects and produced rapid onset of therapeutic action and much improved bioavailability [3].

\section{History of Nanotechnology in Drug Delivery}

The idea of nanotechnology was initially proposed by Feynman in 1959. The term nanotechnology was first devised by Norio Taniguchi in the year 1974 [4] whereas the term nano-medicine was coined by Drexler and colleagues in 1980's [5]. The first development in the field was reported in early 70s at the ETH Zurich, when the first controlled released system was developed along with other several drugs that showed the capability to cross the blood brain barrier with much improved pharmacokinetics [6]. However, it wasn't until 2006 when the first international journal in nano-medicine was introduced called 'International Journal of Nano-medicine' to cater to the emerging needs of nanotechnology in medicine [7].

\section{Advances in Drug Delivery System through Nanotechnology}

This new innovative technique has had revolutionary impact on the management of prevalent diseases.

\section{Cancer}

According to a report published in 2013 an average of 1,660,290 fresh cancer cases were reported to occur in the United States alone in that year [8]. Another report published in the same year [9], segregated the types of cancer according to the most prevalent one in accordance to the geographical region. Cervix cancer was highly reported in Africa and prostate cancer in North America, whereas stomach cancer was highly recognized in Eastern Asia [9]. The staggering numbers of patients suffering from cancer only emphasized the need for development of a novel drug delivery system with enhanced specificity, improved therapeutic efficacy and decreased side effects.

The ability of the drug to reach their objective location and minimum action at non-specific sites determines the superiority of anti-cancer treatments [10]. This could only be achieved by modification at the surface of the nano particles carriers. Incorporation of nano particles with Poly Ethylene Glycol (PEG)/ Poly Ethylene Oxide (PEO) not only reduces non-specific uptake but also enhances specific tumor-targeting ability by preventing white blood cells from identifying the nano particles as foreign objects and allowing them to flow in the blood stream extended enough to attach to tumors [11]. The prime example of this avant-garde technology was the introduction of hydro gel, which when administered by subcutaneous route allows drug delivery for weeks with a single dose [12]. Herceptin, which is presently accepted as an intravenous therapy with a loading dose followed by 6 mg.kg-1 or 2 mg.kg ${ }^{-1}$ every 3 weeks or every week respectively. It acts by targeting cancer cells that display HER2+ (human epidermal growth factor receptor 2), which exists in about one of every four breast cancer patients and makes this type of breast cancer more aggressive than any other type of breast cancer $[13,14]$. After the formulation of hydro gel a team of researchers have succeeded in designing a vitamin E-based hydrogel that can release Herceptin under the skin for several weeks [15]. It has been observed that this hydrogel possesses greater antitumor ability in comparison to conventional intravenous and subcutaneous delivery of Herceptin, due to better reservation of compound within the tumor [15].

Another approach to improve the capability of drug to enter tumors is the use of photosensitizing agents, where the photosensitizing agent

*Corresponding author: Safila Naveed, Faculty of Pharmacy, Jinnah University for Women, Karachi, Pakistan, Tel: 00923002621917; E-mail: safila117@yahoo.com

Received October 27, 2016; Accepted November 25, 2016; Published November 30, 2016

Citation: Maroof K, Zafar F, Ali H, Naveed S, Tanwir S (2016) Scope of Nanotechnology in Drug Delivery. J Bioequiv Availab 8: 001-005. doi:10.4172/ jbb.1000257

Copyright: ( 2016 Maroof K, et al. This is an open-access article distributed under the terms of the Creative Commons Attribution License, which permits unrestricted use, distribution, and reproduction in any medium, provided the original author and source are credited. 
is caused to gather in the tumor, and then the tumor is irradiated with light of suitable wavelength. The photosensitizing agent facilitates the blood vessels in the tumor to become highly permeable, therefore allowing more drug carrying nano particles to enter the tumor. Novel ceramic-based nanoparticles known as Ultrafine has been reported which is a modified silica-based nanoparticles. It exhibits its action by entrapping water-insoluble photosensitizing anticancer drug 2-devinyl2-(1-hexyloxyethyl) pyropheophorbide. In vitro studies have confirmed the active uptake of drug-doped nanoparticles into the cytosol of tumor cells produces significant destruction [16].

Nano disks (NDs) are nano scale, disk-shaped phosphor lipid bi-layers whose edge is stabilized by apolipoproteins [17] and can be developed in vitro by combining recombinant apoA-I proteins with different phospholipids [18]. A study showed that by using laponite (LAP) nanodisks effective transport of doxorubicin (DOX) to cancer cells can take place [19]. Ion exchange process was used to encapsulate DOX into the interlayer space of LAP, in this study the results showed a greater therapeutic efficacy in hindering the growth of a model cancer cell line than free DOX drug at the similar concentration [19].

Super-para-magnetic iron oxide nano particles (SPION) have been widely used experimentally for drug delivery. Magnetic nanoparticles can bind to drugs and can be directed towards tumor using an external magnetic field. Absorption, distribution, metabolism and excretion of the drug are dictated by the nature of surface coating, surface chemistry and size of nanoparticles [20]. A group of researchers have formulated water-dispersible oleic acid (OA)-Pluronic-coated iron oxide magnetic nanoparticle product that not only provides an advantage of loading high doses of water-insoluble anticancer agents but can also be employed as a general drug carrier system for systemic administration of class II drugs while simultaneously allowing magnetic targeting and/ or imaging [21]. The nano particles showed an additional advantage of sustained release and a sustained intracellular drug withholding as compared to drug in solution while exhibiting an anti-proliferative effect in different types of tumors [21].

Another approach to target cancer cells is conveying conjugated gold nano particles with specific peptides to the nuclei of cancer cells. The gold nano particles confine at the nucleus of a cancer cell and impair the DNA due to the failure to thorough cell division resulting in apoptosis [22].

Carbon nanotubes have found great importance in drug delivery of chemotherapeutic agents since 2004 because of their potential to minimize the side effects [23]. Detailed research was performed to calculate approximately the opposite radius of the nano tube that will provide the maximum uptake of the drug molecule [24]. Cisplastin was used as a model drug, which is the first platinum based drug approved to be used in clinics about 40 years ago [25]. Cisplatin acts as a DNAdamaging agent and brings about cells to arrest at either the G1-, S- or G2-phase of the cell cycle in an effort to heal the damage. Weakening satisfactory repair, the cells ultimately experience an abnormal mitosis followed by apoptosis [26]. Three orientations of cisplatin were examined for nano tubes. It was revealed that, for all three orientations of cisplatin to be recognized into the carbon nanotube, the least radius must be at least $4.785 \AA$. This research laid the foundation for an essential pilot design which might offer cancer researchers complete guiding principle to ensure the efficient working of these nano tubes [24]. Another study [27] premeditated the effects of using carbon nano tubes immersed in poly ethylene glycol (PEG). Paclitexal (PTX) was used as a model drug here. The samples showed greater effectiveness to destroy HeLa cells and MCF-7 cells, than free PTX. Hence, these showed great capability to be employed for cancer therapeutics [27].

Carbon nano fibers (CNF's) were formed and discovered in the course of catalytic transformation of carbon-containing gases. These days CNF's are used for various biomedical applications [28]. Polyurethane (PU) is a multipurpose elastomer that is usually employed in biomedicine [29]. A group of scientists examined the dispersion of CNFs in PU with varying PU and CNF weight ratio to make an ideal bladder prosthetic material that should not only encourage usual chief human urothelial cell (HUC) purpose, but also hinder the reappearance of bladder cancerous cell action. The results were promising and delivered a new nano composite comprising of CNF and PU that should be considered for preventing the reoccurrence of cancerous bladder tissue and for encouraging usual non-cancerous bladder tissue development [30-36].

Number of studies have been reported in past few years related to the efficacy and safety of various nanoparticles like cholesteroltethered platinum II-based supramolecular nanoparticle [37-41], polymeric-ceramic nanocarriers (NCs) in order to achieve oral delivery of the anticancer neutraceutical iron-saturated bovine lactoferrin (Fe-bLf) protein) [42,43], novel alginate-enclosed chitosan-calcium phosphate-loaded iron-saturated bovine lactoferrin nanocarriers for oral delivery in colon cancer therapy etc. [44-50]. Another targeting systems for cancer treatment was also investigated with microRNAdirected oncology approach using MRX34 (a liposome-containing miRNA carrier), for usefulness in metastatic liver cancer [51,52]. The amalgamation of various targeted and control release polymeric nano particles have also been illustrated with different programmable approaches and applications. One of them is nanotherapeutic product of docetaxel (BIND-014), which is tested for its effectiveness in solid tumors [53].

\section{Cardiovascular Disease}

Cardiovascular diseases may produce a negative impact not only on economy but also on the quality of life, hence there is an urgent need to develop more effective approaches for the prevention and management of CHF [31]. The burden of stroke is high and is expected to further upturn in forth coming years as a consequence of demographic and epidemiological changes in populations [32]. Hypertension is an imperative community health challenge [33]. Blockade of standard blood movement, is one of the foremost cause of death and prolonged disability [34].

A new nano therapeutic approach has the potential to advance both the effectiveness and efficacy of thrombolytic drugs. It involves the mechanical activation within blood vessels by high-fluid shear strains to selectively aim drugs to locations of vascular hindrance. In vitro and in vivo readings have revealed that this methodology can be employed competently to lyse clots by means of a significantly lesser quantity of thrombolytic drug than is a prerequisite when given in a soluble preparation [34]. The prime example of this is seen in application of dendrimers. Dendrimers are circular designed nanoparticles that have been considered as a mean to administer medicinal agents for a variety of diseases [35]. A group of researchers developed a technique to attach tissue plasminogen activator (tPA) to dendrimers creating a drug delivery system which would necessitate lesser doses with reduced side effects. The results revealed that this drug delivery system produced an advanced concentration of tPA- dendrimer complex during the course of a varied proportion of dilutions [36].

Nanoburrs technique has also been utilized by the researchers to 
combat cardiovascular diseases. These are the nanoparticles covered with an adhesive protein that makes them adhere to arterial walls while they gradually discharge medications [37].

\section{Opthamology}

Even though eye drops and drug-saturated lenses ensure local effect but their use is rendered inadequate due to loss of drug by eruption from eye and also due to absence of prolonged therapeutic delivery. A group of scientists formulated a nanodiamond (ND)-embedded contact lens having the ability of lysozyme-triggered release of drugs for persistent therapy [38], here Timolol maleate was used as a model drug and the results showed that ND-embedded lenses provided prolonged delivery of TM making them promising tools for ocular drug delivery [38]. Ibrahim et al. in 2015 formulated and tested the biodegradable nanoparticles with natural bioadhesives for ophthalmic application of Brimonidine in glaucoma management. Nanoparticles were illustrated with respect to particle size, morphology, zeta potential \& content of drug [54-56]. Nano particles of Celecoxib-loaded drug were developed using different biodegradable polymers like poly(L-lactide) (PLA), poly- $\varepsilon$-caprolactone (PCL) and poly(D,L-lactide-co-glycolide) (PLGA) by impulsive solvent emulsification of diffusion process. Release of drug from these formulations was exhibited in sustained way with no bursting effect. Non-fickian diffusion with suitable fitting to Higuchi model was observed. Toxicity potential of these products was in acceptable level, when determined by cytotoxic examination [57].

\section{Orthodontology and Orthopedics}

Research has proved that nanotechnology has a great scope in the field of onthrodontology and orthopedics. Investigators are refining dental implants by the addition of nano tubes to the outward of the implant. It increases the capability to load the nanotubes with antiinflammatory drugs that can be applied directly to the area around the implant [39]. Silver nanoparticles have gained an ever increasing attention in orthopaedics due to their promising antimicrobial features. They are utilized in tumour prostheses, trauma implants and also as bone cement [58]. In another study novel defensive salivary peptides \& the chitosan based nanoparticles as delivery system were studied extensively in orthodontic field [59].

\section{Diabetes}

Scientists reported that nanoparticles illustrate distinctive biophysical features which are estimated by their size and shape [60]. Nanoparticles depend upon the characteristics of the active compound and as carriers for vaccine delivery with the development of microand nano-technologies; several micro-engineering methods have been developed to fabricate three-dimensional (3D) islet models in vitro which can better mimic the islet of pancreases in vivo. These in vitro islet models have shown better cell function than monolayer cells, indicating their great potential as better experimental platforms to elucidate islet behaviors under both physiological and pathological conditions, such as the molecular mechanisms of diabetes and clinical islet transplantation.

The use of chitosan in drug delivery has two-fold purpose. Scientists demonstrated the usage of chitosan reduced gold nanoparticles as carriers for drug delivery of insulin and the outcomes showed that oral and nasal administration of insulin loaded chitosan reduced gold nanoparticles has resulted in improved pharmacodynamic effect encompassing the ability to manage the postprandial hyperglycemia [40].

Another novel approach utilizing nanotechnology to treat hyperglycemia is a self-regulating glucose-responsive closed-loop insulin delivery system. Scientists reported that microgels with enzyme nanocapsules enables insulin discharge and causes a decrease in blood glucose levels in a mouse model of Type I diabetes. The microgel system expanded when exposed to hyperglycemic environments acting as a regulator and releasing insulin as a consequence of the enzymatic transformation of glucose into gluconic acid and protonation of the chitosan network [41]. Scientists developed various micro-engineering techniques which is utilized to fabricate in vitro $3 \mathrm{D}$ (three-dimensional) islet models which can facilitate islet of pancreases in vivo under different physiological and pathological conditions i.e., clinical islet transplantation and different molecular mechanisms of diabetes [61]. Lee and Kim [62] construct biomimetic nano-sized polymers with bioreducible poly (disulfide amine)s for the management of anemia, cancer, cardiovascular disease and diabetes.

\section{Lung Diseases}

Another potential application of nanoparticles is in investigative and therapeutic aiming of diagnostic material's transport to the lungs. It has been reported that nanoparticles have established an extraordinary retaining power in lung airspaces and alveolar macrophages [42]. Confocal microscopy was applied in one study to display the ardent intake of mesoporous silica nanoparticles labeled as RITC and loaded with isoniazid (anti-tubercular drug) by macrophages of human [54] These macrophages are infected with Mycobacterium tuberculosis GFP-expression. Mohseni et al. in 2015 also assessed the probability of rifampin loading into mesoporous nanoparticles of silica such nanoparticles were found to be proficient in releasing drug contents about $95 \%$ in 24 hours [55].

\section{Inflammation}

Nanoparticles-mediated delivery of antimicrobial agent(s) into macrophages is probable therapeutic objectives in many human ailments $[43,44]$. Polyalkylcyanoacrylates (PACA) nanoparticles were employed as a transporter for directing anti-leishmanial drugs into macrophages. This nano material did not encourage interleukin-1 discharge by macrophages [45]. Another group of scientists add the antifungal and anti-leishmanial agent amphotericin $\mathrm{B}(\mathrm{AmB})$ and complexed with nanotubes to develop a less toxic formulation in trilaurin based nanosize lipid particles (emulsomes) stabilized by soya phosphatidylcholine as a new intravenous drug delivery system for macrophage targeting [46]. Nanoemulsions of anti-inflammatory agent Flurbiprofen have also been stated in literature [47]. Also, nanoaAPC stimulation enhanced by magnetic field can develop massive numbers of activated antigen-specific $\mathrm{T}$ cells which can be used for adoptive immunotherapy [63]. Various categories of nanomaterials i.e., nanoporous scaffolds, nanoparticles, carbon nanotubes and nanofibers are widely utilized for various processing techniques. These nanomaterials are efficiently used in the field of tissue engineering to enhance the manufacturing of biomimetic alternates to substitute damaged organs and tissues. Applications of nanomaterials in tissue engineering are dependent upon the inflammatory conditions they elicit in vivo [64].

\section{Conclusion}

The current advancement in the delivery of water-insoluble drugs and biopharmaceuticals are expected to notably influence the pharmaceutical and biotechnological industries. Furthermore, the relentless advancement in the current drug delivery nanotechnology 
which scientists could use to develop formulations has had a remarkable impact on reducing cost and improving the efficiency and productivity.

\section{References}

1. Ochekpe NA, Olorunfemi PO, Ngwuluka NC (2009) Nanotechnology and Drug DeliveryPart 1: Background and Applications. Trop J Pharm Res 8: 265-274.

2. Jain N, Jain R, Thakur N, Gupta BP, Jain DK, et al. (2010) Nanotechnology: A Safe and effective drug delivery system. Asian J Pharm Clin Res 3: 159-165.

3. Kakade T, Kadam V, Dhanavade K, Salunkhe V (2013) A review on pharmaceutical nanotechnology: dendrimers. WJPPS 2: 4815-4830.

4. Webster TJ (2007) IJN's second year is now a part of nanomedicine history! Int J Nanomedicine 2: 1-2.

5. Tibbals HF (2011) Medical Nanotechnology and Nanomedicine. CRC press Taylor and Francis group 31

6. Najlah M, Freeman S, Attwood D, D'Emanuele A (2007) In vitro evaluation of dendrimer prodrugs for oral drug delivery. Int J Pharm 336: 183-190.

7. Cevc G (2004) Lipid vesicles and other colloids as drug carriers on the skin. Adv Drug Deliv Rev 56: 675-711.

8. Siegel R, Naishadham D, Jemal A (2013) Cancer statistics, 2013. CA Cancer J Clin 63: 11-30.

9. Bray F, Ren JS, Masuyer E, Ferlay J (2013) Global estimates of cancer prevalence for 27 sites in the adult population in 2008. Int J Cancer 132: 11331145.

10. van Vlerken LE, Vyas TK, Amiji MM (2007) Poly(ethylene glycol)-modified nanocarriers for tumor-targeted and intracellular delivery. Pharm Res 24: 14051414.

11. Biswas AK, Islam R, Choudhury ZS, Mostafa A and Kadir MF (2014) Nanotechnology based approaches in cancer therapeutics. Adv Nat Sci: Nanosci Nanotechnol 5: 2043-6262.

12. Gupta P, Vermani K, Garg S (2002) Hydrogels: from controlled release to $\mathrm{pH}$ responsive drug delivery. Drug Discov Today 7: 569-579.

13. McKeage K, Lyseng-Williamson KA (2008) Trastuzumab: a pharmacoeconomic review of its use in early breast cancer. Pharmacoeconomics 26: 699-719.

14. Baselga J (2000) Current and planned clinical trials with trastuzumab (Herceptin). Semin oncol 27-32

15. Steinbach OC (2014) Industry Update: the latest developments in therapeutic delivery. Ther Deliv 5: 505-509.

16. Roy I, Ohulchanskyy TY, Pudavar HE, Bergey EJ, Oseroff AR, et al. (2003) Ceramic-based nanoparticles entrapping water-insoluble photosensitizing anticancer drugs: a novel drug-carrier system for photodynamic therapy. J Am Chem Soc 125: 7860-7865

17. Ghosh M, Singh AT, Xu W, Sulchek T, Gordon LI, et al. (2011) Curcumin nanodisks: formulation and characterization. Nanomedicine 7: 162-167.

18. Murakami T (2012) Phospholipid nanodisc engineering for drug delivery systems. Biotechnol J 7: 762-767.

19. Wang S, Wu Y, Guo R, Huang Y, Wen S, et al. (2013) Laponite nanodisks as an efficient platform for Doxorubicin delivery to cancer cells. Langmuir 29: 5030-5036.

20. Gupta AK, Gupta M (2005) Synthesis and surface engineering of iron oxide nanoparticles for biomedical applications. Biomaterials 26: 3995-4021.

21. Jain TK, Morales MA, Sahoo SK, Leslie-Pelecky DL, Labhasetwar V (2005) Iron oxide nanoparticles for sustained delivery of anticancer agents. Mol Pharm 2: 194-205.

22. Kang B, Mackey MA, El-Sayed MA (2010) Nuclear targeting of gold nanoparticles in cancer cells induces DNA damage, causing cytokinesis arrest and apoptosis. J Am Chem Soc 132: 1517-1519.

23. Chen J, Chen S, Zhao X, Kuznetsova LV, Wong SS, et al. (2008) Functionalized single-walled carbon nanotubes as rationally designed vehicles for tumortargeted drug delivery. J Am Chem Soc 130: 16778-16785.

24. Hilder TA, Hill JM (2008) Theoretical comparison of nanotubes materials for drug delivery. Micro Nano Lett 3: 18-24.
25. Desoize B, Madoulet C (2002) Particular aspects of platinum compounds used at present in cancer treatment. Crit Rev Oncol Hematol 42: 317-325.

26. Eastman A (1991) Mechanisms of resistance to cisplatin. Cancer Treat Res 57: $233-249$

27. Liu Z, Sun X, Nakayama-Ratchford N, Dai H (2007) Supramolecular chemistry on water-soluble carbon nanotubes for drug loading and delivery. ACS Nano 1: $50-56$.

28. Serp P, Corrias M, Kalck P (2003) Carbon nanotubes and nanofibers in catalysis. Applied Catalysis A: General 253: 337-358.

29. Romaškevic T, Budriene S, Pielichowski K and Pielichowski J (2006) Application of polyurethane-based materials for immobilization of enzymes and cells: a review. Chemija 17: 74-89.

30. Tsang M, Chun YW, Im YM, Khang D, Webster TJ (2011) Effects of increasing carbon nanofiber density in polyurethane composites for inhibiting bladder cancer cell functions. Tissue Eng Part A 17: 1879-1889.

31. Rich MW (1997) Epidemiology, pathophysiology, and etiology of congestive heart failure in older adults. J Am Geriatr Soc 45: 968-974.

32. Heidenreich PA, McClellan M (2001) Trends in treatment and outcomes for acute myocardial infarction: 1975-1995. Am J Med 110: 165-174.

33. Kearney PM, Whelton M, Reynolds K, Whelton PK, He J (2004) Worldwide prevalence of hypertension: a systematic review. J Hypertens 22: 11-19.

34. Korin N, Gounis MJ, Wakhloo AK, Ingber DE (2015) Targeted drug delivery to flow-obstructed blood vessels using mechanically activated nanotherapeutics. JAMA Neurol 72: 119-122.

35. Wolinsky JB, Grinstaff MW (2008) Therapeutic and diagnostic applications of dendrimers for cancer treatment. Adv Drug Deliv Rev 60: 1037-1055.

36. D'Emanuele A, Attwood D (2005) Dendrimer-drug interactions. Adv Drug Deliv Rev 57: 2147-2162.

37. Lee L, Horowitz J, Frenneaux M (2004) Metabolic manipulation in ischaemic heart disease, a novel approach to treatment. Eur Heart J 25: 634-641.

38. Hiratani H, Fujiwara A, Tamiya Y, Mizutani Y, Alvarez-Lorenzo C (2005) Ocular release of timolol from molecularly imprinted soft contact lenses. Biomaterials 26: 1293-1298.

39. Bjursten LM, Rasmusson L, Oh S, Smith GC, Brammer KS, et al. (2010) Titanium dioxide nanotubes enhance bone bonding in vivo. J Biomed Mate Res A 92: 1218-1224.

40. Bhumkar DR, Joshi HM, Sastry M, Pokharkar VB (2007) Chitosan reduced gold nanoparticles as novel carriers for transmucosal delivery of insulin. Pharm Res 24: $1415-1426$.

41. Gu Z, Dang TT, Ma M, Tang BC, Cheng H, et al. (2013) Glucose-responsive microgels integrated with enzyme nanocapsules for closed-loop insulin delivery. ACS Nano 7: 6758-6766.

42. Sajja HK, East MP, Mao H, Wang YA, Nie S, et al. (2009) Development of multifunctional nanoparticles for targeted drug delivery and noninvasive imaging of therapeutic effect. Curr Drug Discov Technol 6: 43-51.

43. Singh RK, Srivastava A, Gour JK, Tiwari VK (2012) Targeting Leishmania Species: Nanotechnological Prospects. Adv Sci Lett 5: 11-20.

44. Gaspar R, Préat V, Opperdoes FR, Roland M (1992) Macrophage activation by polymeric nanoparticles of polyalkylcyanoacrylates: activity against intracellula Leishmaniadonovani associated with hydrogen peroxide production. Pharm Res 9: 782-787.

45. Balland O, Pinto-Alphandary H, Viron A, Puvion E, Andremont A, et al. (1996) Intracellular distribution of ampicillin in murine macrophages infected with Salmonella typhimurium and treated with $(3 \mathrm{H})$ ampicillin-loaded nanoparticles. J Antimicrob Chemother 37: 105-115.

46. Gupta S, Vyas SP (2007) Development and characterization of amphotericin $\mathrm{B}$ bearing emulsomes for passive and active macrophage targeting. J Drug Target 15: 206-217.

47. Maroof K, Zafar F, Ali H and Naveed S (2015) Flurbiprofen: A Potent Pain Reliever. J Bioequiv Availab 7: 56-58.

48. Sengupta P, Basu S, Soni S, Pandey A, Roy B, et al. (2012) Cholesteroltethered platinum II-based supramolecular nanoparticle increases antitumo efficacy and reduces nephrotoxicity. Proc. Natl Acad Sci 109: 11294-11299. 
Citation: Maroof K, Zafar F, Ali H, Naveed S, Tanwir S (2016) Scope of Nanotechnology in Drug Delivery. J Bioequiv Availab 8: 001-005. doi:10.4172/ jbb.1000257

49. Kanwar JR, Mahidhara G, Kanwar RK (2013) Novel alginate-enclosed chitosancalcium phosphate-loaded iron-saturated bovine lactoferrin nanocarriers for oral delivery in colon cancer therapy. Nanomedicine 7: 1521-1550.

50. Lee JM, Yoon TJ, Cho YS (2013) Recent developments in nanoparticle-based siRNA delivery for cancer therapy. Biomed Res Int 2013: 782041.

51. (2013) A Multicenter Phase I Study of MRX34, MicroRNA miR-RX34 Liposome Injectable Suspension.

52. Bader AG (2012) miR-34 - a microRNA replacement therapy is headed to the clinic. Front Genet 3: 120.

53. Sanna V, Pala N, Sechi M (2014) Targeted therapy using nanotechnology: focus on cancer. Int J Nanomedicine 9: 467-483.

54. Hwang AA, Lee BY, Clemens DL, Dillon BJ, Zink JI, et al. (2015) Tuberculosis: $\mathrm{pH}$-Responsive Isoniazid-Loaded Nanoparticles Markedly Improve Tuberculosis Treatment in Mice. Small 11: 50-65.

55. Mohseni M, Gilani K, Mortazavi SA (2015) Preparation and characterization of rifampin loaded mesoporous silica nanoparticles as a potential system for pulmonary drug delivery. Iran J Pharm Res 14: 27-34.

56. Ibrahim MM, Abd-Elgawad AH, Soliman OA, Jablonski MM (2015) Natural Bioadhesive Biodegradable Nanoparticle-Based Topical Ophthalmic Formulations for Management of Glaucoma. Transl Vis Sci Technol 4: 12.
57. Ibrahim MM, Abd-Elgawad AE, Soliman OA, Jablonski MM (2013) Nanoparticlebased topical ophthalmic formulations for sustained celecoxib release. J Pharm Sci 102: 1036-1053.

58. Brennan SA, NÃ FhoghlÃ ${ }^{\circ} \mathrm{C}$, Devitt BM, O'Mahony FJ, Brabazon D, et al. (2015) Silver nanoparticles and their orthopaedic applications. Bone Joint J 97-97B: 582-589.

59. Zaidan CSF (2015) Novel protective salivary peptides and the chitosan nanoparticles delivery system. Electronic Thesis and Dissertation Repository. Paper 3159.

60. Jeevanandam J, Danquah MK, Debnath S, Meka VS, Chan YS (2015) Opportunities for Nano-Formulations in Type 2 Diabetes Mellitus Treatments. Curr Pharm Biotechnol 16: 853-870.

61. Gao B, Wang L, Han S, Pingguan-Murphy B, Zhang X, et al. (2015) Engineering of microscale three-dimensional pancreatic islet models in vitro and their biomedical applications. Crit Rev Biotechnol 15: 1-11.

62. Lee YS, Kim SW (2014) Bioreducible polymers for therapeutic gene delivery. J Control Release 190: 424-439.

63. Perica K, Tu A, Richter A, Bieler JG, Edidin M, et al. (2014) Magnetic fieldinduced $T$ cell receptor clustering by nanoparticles enhances $T$ cell activation and stimulates antitumor activity. ACS Nano 8: 2252-2260.

64. Padmanabhan J, Kyriakides TR (2015) Nanomaterials, inflammation, and tissue engineering. Wiley Interdiscip Rev Nanomed Nanobiotechnol 7: 355-370. 